Background Lupus is an autoimmune disease characterized by antibodies directed against nuclear components that induce immune complex-mediated injury to multiple organs. Underlying lupus is the induction of T-cell-dependent activation and clonal expansion of autoreactive $\mathrm{B}$ cells in germinal centers resulting in their differentiation into plasma cells that secrete pathogenic autoantibodies. Heightened glucose metabolism is inherent to immune/inflammatory disorders, but little is known of its role in lupus etiology. Present treatments for lupus rely heavily on broad-spectrum immunosuppressive agents, and there is a need for targeted therapies that effectively counteract this systemic autoimmune disorder.

Methods Here we examined the metabolic and gene expression profiles of key autoimmune populations in mouse models of spontaneous lupus and their responses to treatment with the glycolysis inhibitor 2-deoxyglucose (2DG) in drinking water. Therapeutic efficacy in terms of survival after long- vs. short-term 2DG-exposed times was assessed on BXSB.Yaa and NZBWF1 lupus-prone mice. Furthermore, a chimeric antigen receptor (CAR)-T immunotherapy was used to determine whether a targeted removal of an identified B cell subset can improve disease outcomes.

Results We found greater glucose uptake and glycolysis rate in spontaneous germinal center B cells (GCB) compared to that in follicular helper $\mathrm{T}$ cells (Tfh). The differential dependency on glucose oxidation between GCB and Tfh was determined, rendering GCB highly susceptible to oxidative stress-induced apoptosis triggered by glycolysis inhibition via 2DG. Short-term glycolytic inhibition selectively targeted GCB, sparing other autoreactive populations, including Tfh. This reduction of GCB is, in turn, responsible for improving kidney function and lifespan of treated mice. Moreover, we identified a subset of GCB, which express TNFSF17 and exhibit a higher reliance on glucose metabolism than TNFSF17 ${ }^{-}$GCB. Their depletion through its ligand TNFSF13-based CAR-T treatment significantly decreased mortality from lupus.

Conclusions Differential metabolic requirement for glucose between autoreactive GCB and Tfh dictates different sensitivity to apoptosis via glycolytic inhibition, and this provides a metabolic niche for targeted lupus treatment. Combining therapies that selectively dampen GCB metabolism for survival with $\mathrm{T}$ cell-based immunotherapy could provide new effective treatments for lupus.

Acknowledgments We thank the funding resources from JAX Director Initiative Fund, the RILITE Foundation, and the John and Marcia Goldman Foundation.

\section{THE EIF4 TRANSLATIONAL INHIBITOR PATEAMINE A IMPROVES IMMUNOLOGICAL AND NEUROLOGICAL FUNCTIONS IN BXSB.YAA LUPUS MICE}

${ }^{1}$ Gonzalo Gómez-Hernández, ${ }^{1}$ Nieves Varela, ${ }^{2}$ Harini Bagavant, ${ }^{1}$ Guillermo Barturen, ${ }^{1}$ María Morell, ${ }^{1}$ Marta E Alarcón-Riquelme*. ' $G e n y o$, Granada, Spain; ${ }^{2}$ OMRF, Oklahoma City, USA

\subsection{6/lupus-2021-lupus21century.80}

Background Systemic Lupus Erythematosus (SLE) is an autoimmune disease characterized by loss of tolerance and activation of the immune response. Clinical manifestations are heterogeneous and several organs can be affected including skin, joints, central nervous system and kidney. Traditional treatments include the use of hydroxychloroquine, glucocorticosteroids, immunosuppressive and more recently, biological drugs such as belimumab or rituximab. In the last decade new alternatives have been proposed based on targeting interferon and cytokines. Mouse models have been extremely helpful to test the efficacy of new SLE therapies. In this work we analyze the therapeutic potential of a natural compound, Patemina A (PatA) to treat SLE. Pat A is an inhibitor of the translation initiation process with immunosuppressive properties that has been tested successfully in cancer mouse models.

Methods To test Pat A efficiency in SLE we used the BXSB. Yaa lupus model. Animals were treated for 8 weeks starting at the initial stages of disease (12 weeks). Sera was collected every three weeks and disease signs were followed. At the final point we performed serological analyses (cytokines and autoantibodies), flow cytometry on spleen to evaluate different cell populations, kidney histological and functional assays and behavioral tests to evaluate neuropsychiatric changes.

Results Our data shows that Pat A treatment increases the survival rate and is able to reduce circulating levels of proinflammatory cytokines and autoantibodies. We also observed improvement of cognitive functions (learning/memory, and depression behavioral tests) together with a reduction of proinflammatory cytokines locally in the hippocampus.

Conclusion These data suggests that translation inhibition improves lupus disease signs at the immunological and neurological levels opening a new line of research based on translation inhibition to treat lupus and possibly other autoimmune diseases with an inflammatory component, and may be a complement for present day treatments.

\section{A PEPTIDE MIMETIC OF THE KINASE INHIBITORY REGION OF SUPPRESSOR OF CYTOKINE SIGNALING-1 ATTENUATES LYMPHOCYTE ACTIVATION AND LUPUS PROGRESSION IN MRL/LPR LUPUS MODEL}

'Jatin Sharma, ${ }^{1}$ Teresa D Collins, ${ }^{2}$ Tracoyia Roach, 'Shiwangi Mishra, ${ }^{1}$ Brandon K Lam, 'Zaynab Sidi Mohamed, 'Antia E Veal, 'Timothy B Polk, 'Amari Jones, ${ }^{1}$ Caleb Cornaby, ${ }^{1}$ Mohammed I Haider, ${ }^{2}$ Leilani Zeumer-Spataro, ${ }^{1}$ Howard M Johnson, ${ }^{2}$ Laurence M Morel, 'Joseph Larkin*. 'Department of Microbiology and Cell Science, University of Florida, Gainesville, FL, USA; '2Department of Pathology, Immunology, and Laboratory Medicine, University of Florida, Gainesville, FL 32610, USA

10.1136/lupus-2021-lupus21century.81

Background Lupus progression is driven by the aberrant activation of $\mathrm{T}$ and $\mathrm{B}$ lymphocytes which promote a dysregulated cytokine milieu and produce pathogenic antibodies. Many cytokines, including interferon gamma (IFN- $\gamma$ ), utilize the janus kinase/signal transduction and activation of transcription (JAK/STAT) pathway for signal propagation. Suppressor of Cytokine Signaling-1 (SOCS-1) is an inducible, intracellular protein which regulates the JAK/STAT pathway and IFN $\gamma$ signaling. We have previously shown that a peptide mimicking the kinase inhibitory region of SOCS1 (SOCS1KIR) inhibited IFN- $\gamma$ signaling and inflammation-mediated disease progression.

Using MRL/lpr mice, which spontaneously develop SLE-like disease, we test the hypothesis that SOCS1-KIR administration inhibits $\mathrm{T}$ and $\mathrm{B}$ lymphocyte activation leading to an amelioration in lupus pathology. 


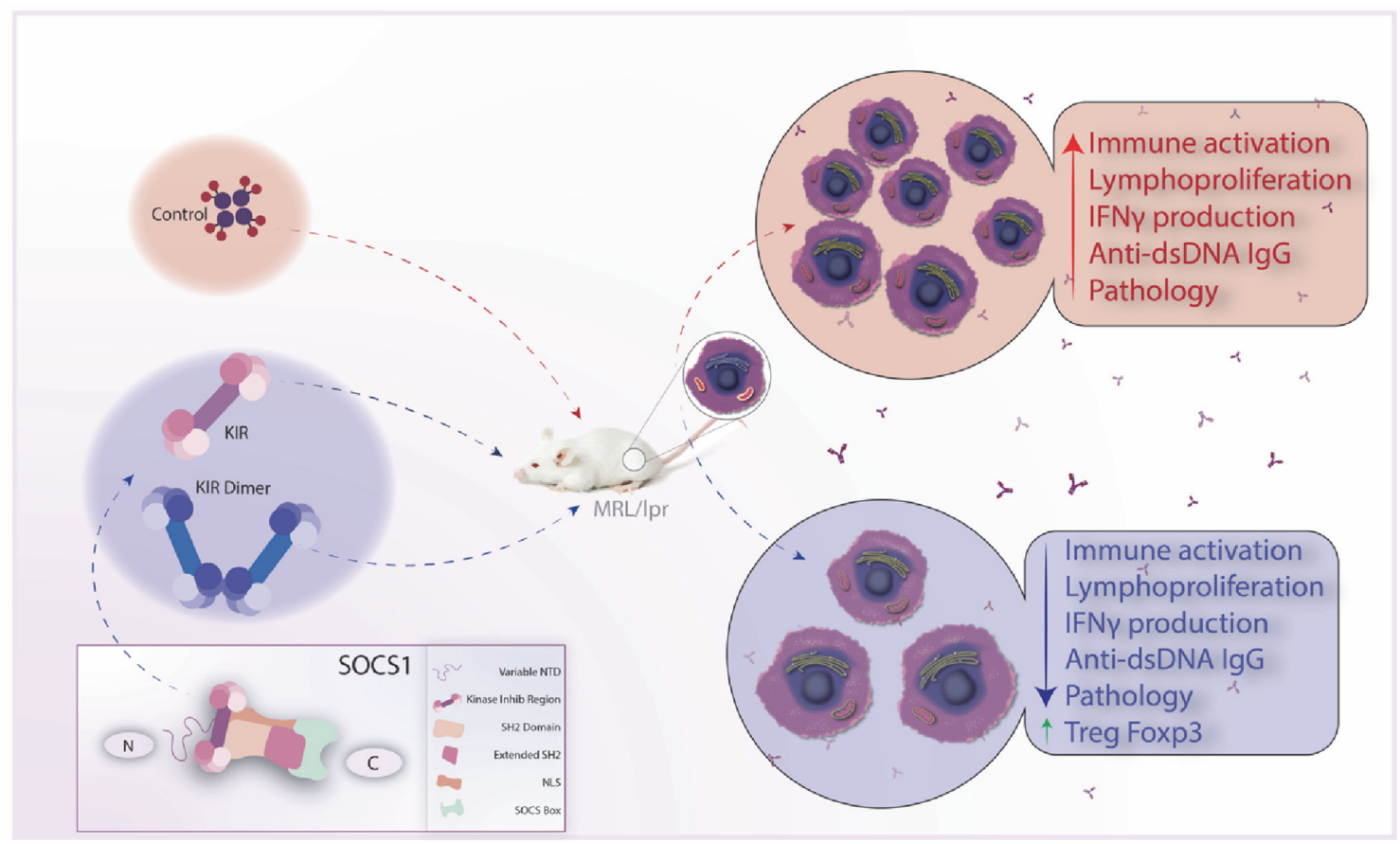

Abstract 1404 Figure 1 Graphical representation of the study design and results

Methods Female MRL/lpr mice received intraperitoneal injections of SOCS1-KIR peptide, or PBS carrier, 3 times per week and were monitored for lupus-like disease progression. Disease progression was based on the presence of skin lesions, lymphadenopathy, overall body score, and proteinuria. Peripheral blood, lymph nodes, and spleen was evaluated for peptide-mediated changes in lymphocyte populations by flow cytometry and qPCR. ELISA and western blot analysis were also employed to assess changes in lymphocyte activation. Finally, peptide mediated changes in renal pathology were analyzed.

Results We show that intraperitoneal administration of SOCS1KIR reduced the frequency, activation, and cytokine production of memory $\mathrm{CD}^{+}$and $\mathrm{CD}^{+} \mathrm{T}$ lymphocytes within the peripheral blood, spleen, and lymph nodes of treated mice. In addition, administration of SOCS1-KIR mimetic peptide treatment reduced lymphadenopathy, delayed the development and severity of skin lesions, reduced autoantibody production, and lupus associated kidney destruction. On a cellular level, SOCS1-KIR administration enhanced Foxp3 expression in both total splenic Tregs and follicular Tregs (figure 1). In addition, SOCS1-KIR treatment reduced the frequency of GL7+ germinal center enriched B cells and CD80+ leukocytes, which may potentially activate $\mathrm{T}$ lymphocytes.

Conclusion Together, these data show that SOCS1-KIR treatment was effective in reducing auto-reactive lymphocyte effector functions and suggest that therapeutic targeting of the SOCS1 pathway through peptide administration may have efficacy in mitigating lupus progression.

Acknowledgement We thank Mr. Benson and Dr. Moneypenny for flow cytometry assistance, UF animal care, and Drs. Hoffman and Wilson for technical expertise. This study was supported by the Lupus Research Institute, National Psoriasis Foundation, a BD Biosciences Research Grant, NIH/NCATS Clinical and Translational Science Awards TL1 TR000066 and UL1TR000064, NIH/NIAID subaward U01AI101990 and The Mcknight Fellowship Foundation.

\section{MECHANISMS UNDERLYING LUPUS SUPPRESSION MEDIATED BY MALE MICROBIOTA TRANSPLANTS IN FEMALE LUPUS-PRONE MICE}

James W Harder, Jing Ma, Pascale Alard, Michele M Kosiewicz*. University of Louisville, Louisville, KY, USA

\subsection{6/lupus-2021-lupus21century.82}

Background Sex and microbiota dysbiosis affect lupus development/progression. We have found that gut microbiota and metabolomic profiles in adult lupus-prone female and lupusresistant male NZBxNZWF1 (BWF1) mice differ; male cecal transplants into female mice suppress disease. Here, we identify disease-modulating bacteria and metabolite candidates and characterize potential mechanisms underlying disease suppression mediated by male microbiota transplants.

Methods BWF1 mice were used for all experiments. Fecal microbiota composition in female and male mice was determined by $16 \mathrm{~S}$ rRNA gene sequencing. Fecal metabolites were identified using mass spectrometry. Cecal contents from adult male or female mice were transplanted by oral gavage into female mice. Disease (proteinuria) and survival, and mesenteric

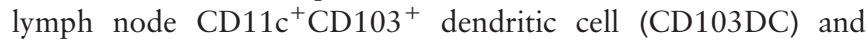
splenic $\mathrm{CD} 11 \mathrm{~b}^{+} \mathrm{F} 4 / 80^{+}$macrophage function in in vitro assays and by RNA-seq/RT-PCR were analyzed. In some experiments, female mice were treated with male fecal metabolites in vivo and cell function analyzed ex vivo, or female cells were treated directly with male metabolites in vitro and function analyzed.

Results Microbiota composition analysis in adult female and male mice and recipients of cecal transplants under conditions when male cecal transplants did or did not suppress disease indicated that high Bacteroides and low Clostridium abundances (high Bacteroides/Clostridium ratio) and presence of Alistipes correlated with disease resistance/suppression. Two types of immune cells with different functions were identified as possible mediators of sex-dependent and microbiota-driven 